\title{
The Correlation Between Elastic Properties and AFM Images of Nanocoatings on Polymers
}

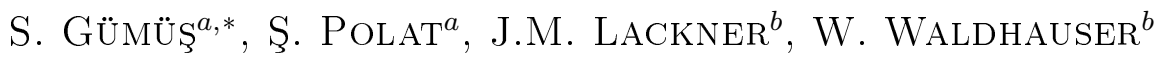 \\ ${ }^{a}$ Kocaeli University, Faculty of Engineering, Department of Metallurgical and Materials Engineering, \\ Umuttepe Campus, 41380 Kocaeli, Turkey \\ ${ }^{b}$ Joanneum Research Forschungsges.m.b.H., Institute of Surface Technologies and Photonics, \\ Functional Surfaces, Leobner Strasse 94, A-8712 Niklasdorf, Austria
}

\begin{abstract}
In a biomimetic approach for designing implants, both the physical properties (such as topography, elasticity, roughness, hydrophilicity, charges etc.) and the chemical structure of the artificial biomaterial should be considered, since they affect the adhesion of proteins in the nanometer-scale and of the cells in micro-scale at the interface. In this case, surface modification of biomaterials plays a major role because of the expectations from the material surface. These may be realized by changing the surface chemistry, physically or chemically, or by coating it with a material having a certain chemical composition. In this study, surface properties like topography and roughness, and mechanical properties like elastic modulus and hardness are determined for nanocoated materials.
\end{abstract}

DOI: 10.12693/APhysPolA.127.1142

PACS: 81.07.-b, 68.55.am, 68.55.J-, 68.37.Ps, 62.20.de, 68.60.Bs

\section{Introduction}

Biomaterials are natural or artificial-based materials used for supporting or replacing a part of the function of a living tissue of human body. In biomaterial applications, apart of the bulk properties of the material, different surfaces are expected, which will come in contact and will interact with organs and tissues. The effects and reactions of biomaterials, experienced in the biological environment, show the complexity of selection and design of these materials. Determination of the mechanical and surface properties of natural biomaterials gives an insight to understand the biological micro- and nanoscale structure and biological function.

Besides metallic and ceramic materials, many polymers are used in various applications due to optimization of physicochemical and biocompatibility properties and manufacturability into complex shapes and structures. However, their surface chemistry is generally not optimized for cell adhesion, and modification of the topography enables biomimetically-based advances. Finally, such polymers for implants in orthopedic surgery, with bulk compliance adapted to bone, fulfill mechanical surface demands for this specific application.

In a biomimetic approach for designing implants, both the physical properties (such as topography, elasticity, roughness, hydrophilicity, charges etc.) and the chemical structure of the artificial biomaterial should be considered, since they affect the adhesion of proteins in nanometer-scale and subsequent adhesion of cells in micro-scale at the interface [1]. In this case, surface mod-

\footnotetext{
* corresponding author; e-mail: sgumus@kocaeli.edu.tr
}

ification of biomaterials plays a major role, because of the expectations from the material surface. These may be realized by changing the surface chemistry physically or chemically, or by coating it with a material having a certain chemical composition.

Surface modification of such coatings by glow discharge, plasma treatment, ion implantation, grafting macromolecules or functional groups etc., is an effective method to improve biological interactions in a desired way, according to the application area. In this way the desired cell attachment and spreading is controlled through the tailored topography and chemistry [2]. Nanometer-scale topography affects the attachment and growth of cells, which is the first step in biomaterial-cell interaction, because the size of protein is of nanometer scale. It has recently been proved that cell adhesion is influenced by surface features as small as $10 \mathrm{~nm}[3,4]$.

Coating polymers by a vacuum processes may cause many problems due to their low thermal stability, and mechanical properties such as elastic modulus and hardness. On the other hand, the difference in the properties of the film and the substrate results in self-assembling surface features in nano-micrometer scale [5], as a result of which biocompatibility is enhanced in terms of protein adsorption. In this study, surface properties like topography and roughness, and mechanical properties like elastic modulus and hardness are determined for nanocoated materials.

\section{Experimental}

The coating process is performed by PVD coating system in Joanneum Research. Titanium (Ti) and titanium nitride (TiN) are deposited on the thermoplastic polyurethane (PU, Advan-Source Biomaterials ChronoThane ${ }^{T M} \mathrm{P}$ ) and polycarbonate (PC, Senova 
Senolex ${ }^{\mathrm{TM}}$ ) at temperatures below $50{ }^{\circ} \mathrm{C}$, with a resulting thickness of $100 \mathrm{~nm}$. Details of the coating conditions are given elsewhere [5]. Additionally, the two inorganic coating materials are deposited on silicon wafer for determination of the hardness and elasticity modulus by the indentation test.

The topographical and mechanical characterization of the coatings is performed by using atomic force microscope (AFM, NanoMagnetics, AmbientAFM) in tapping mode and ultra-micro hardness indentation apparatus (Fischerscope HV100, Berkovich diamond indenter, (load of $1.5 \mathrm{mN}$, loading/unloading rate of $0.2 \mathrm{mN} / \mathrm{s}$ ), respectively. The deformation and fracture behavior of the coatings is examined by indentation test at different loads such as $50 \mathrm{mN}, 100 \mathrm{mN}, 150 \mathrm{mN}, 200 \mathrm{mN}, 250 \mathrm{mN}$ and $300 \mathrm{mN}$, with a constant loading rate of $4 \mathrm{mN} / \mathrm{s}$. The indentation impressions are examined by Jeol, JSM 6060 scanning electron microscope (SEM).

\section{Results and discussion}

Indentation technique allows to characterize the mechanical properties of thin films, coatings, etc. by sensing the load and displacement data during loading and unloading of an indenter to the coating [6]. A great advantage of the indentation test is the direct measurement of the hardness and elastic modulus without imaging the impression of the indentation. Further, the fracture toughness, i.e. the deformation behavior under different loads can be evaluated by examination of the indentation impression using SEM [7].

The hardness and elasticity modulus of the coatings are determined from the indentation test on coated silicon wafer under the same conditions, to eliminate the substrate effect of the soft polymers during indentation procedure. The results are given in Table. As expected, hardness and elasticity modulus of TiN coating are much higher than those of $\mathrm{Ti}$ coating on $\mathrm{Si}$.

Indentation test results.

TABLE

\begin{tabular}{l|c|c|c}
\hline \hline & Ti on $\mathrm{Si}$ & TiN on $\mathrm{Si}$ & $\mathrm{Si}$ \\
\hline Test Load, [mN] & 1.500 & 1.500 & 4 \\
$h_{\text {max }}^{a},[\mu \mathrm{m}]$ & $0.057 \pm 0.003$ & $0.043 \pm 0.001$ & 0.091 \\
$h_{p}^{b},[\mu \mathrm{m}]$ & $0.036 \pm 0.004$ & $0.020 \pm 0.002$ & 0.024 \\
$H,[\mathrm{GPa}]$ & $5.52 \pm 0.41$ & $8.33 \pm 0.47$ & 7.25 \\
$H_{\text {plast. }}[\mathrm{GPa}]$ & $10.78 \pm 1.92$ & $34.52 \pm 4.99$ & 23.59 \\
$E$-modulus, [GPa] & $140.50 \pm 3.6$ & $180.23 \pm 11.11$ & 160.31 \\
Plasticity, $h_{p} / h_{\text {max }}$ & 0.63 & 0.46 & 0.26 \\
${ }^{a}$ Indentation depth at peak load. \\
${ }^{b}$ Final depth of the residual hardness impression.
\end{tabular}

Recently, AFM is widely used for imaging of nanometer-sized surface structures due to its ability of providing high-resolution images in atomic scale. Three dimensional (3D) images of the surface are obtained by $\mathrm{AFM}$, giving information about the surface morphology, roughness, features formed at the surface, their shape and distribution.
The AFM images taken in tapping mode of an area of $1 \times 1 \mu \mathrm{m}^{2}$ of $\mathrm{Ti}$ and $\mathrm{TiN}$ coated $\mathrm{PC}$ and $\mathrm{PU}$ are given in Fig. 1. AFM images of $\mathrm{Ti}$ and TiN differ from each other in terms of morphology. The surfaces coated with $\mathrm{Ti}$ by magnetron sputtering (low energetic deposition) reveal separated, small island-like features and columnar growth with dome-shaped column tops (Fig. 1a and 1b). On the other hand, as seen in Fig. 1c and 1d, the surfaces coated with TiN by the more energetic pulsed laser deposition (PLD), present large vermicular-like, wavy topographies (i.e. wrinkles). The effect of substrate is also visible, different topography is formed on stiff PC and soft PU. PC has quite low elongation at fracture and a high elastic modulus compared to PU. Surface of the stiff $\mathrm{PC}$ is not able to deform at the beginning of the film formation. So the island-like structures, caused by the intrinsic stress, cover uniformly the substrate's surface. Further film growth leads to regular columnar structures [8]. Thus, no wrinkles are seen on $\mathrm{Ti}$ coatings (Fig. 1a and 1b), due to low energetic magnetron sputtered deposition, which causes low intrinsic stress, whereas to form wrinkles, a high intrinsic growth stress is required. In TiN coatings due to their composition (high elastic modulus), there are high enough intrinsic growth stresses, which produce wrinkles (Fig. 1c and 1d). In the TiN-coated PU, those wrinkles are larger in size, compared to the TiN-coated PC sample, since the differences in the mechanical properties of the coating and of the substrate are higher.
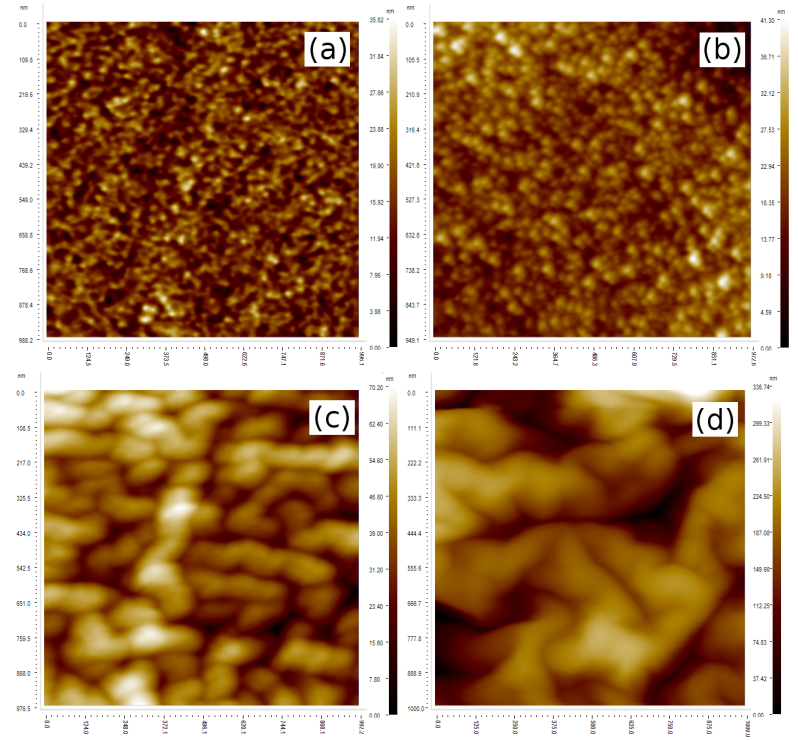

Fig. 1. AFM topographical images of Ti-coated (a) PC, (b) PU, and TiN-coated (c) PC, (d) PU.

Indentation test may be used to evaluate the fracture behavior of the coated materials. After unloading step, the characteristic traces are left on the specimen's surfaces. The cracks appear either at the interface between the coating and the substrate or within the coating itself [9]. The formula below has been proposed to determine the fracture toughness $\left(K_{C}\right)$, using the length of 
radial crack $(c)$, progressing from the corners of sharp indenter,

$$
K_{C}=\alpha\left(\frac{E}{H}\right)^{1 / 2}\left(\frac{P}{c^{3 / 2}}\right),
$$

where, $\alpha$ is a parameter that depends on the geometry of the indenter (for Berkovich it is 0.016), $E$ is elasticity modulus, $H$ is hardness and $P$ is peak indentation load [10].

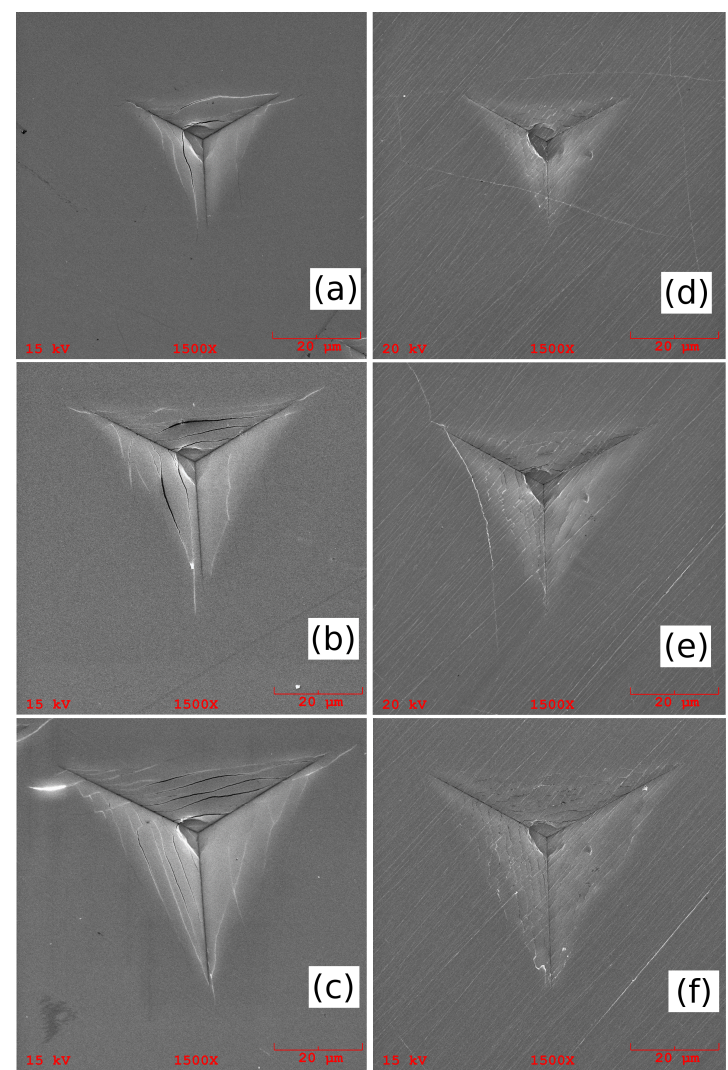

Fig. 2. The SEM images of indentation impressions at loadings (a) $100 \mathrm{mN}$, (b) $200 \mathrm{mN}$, (c) $300 \mathrm{mN}$ of Ticoated PC and (d) $100 \mathrm{mN}$, (e) $200 \mathrm{mN}$, (f) $300 \mathrm{mN}$ of TiN-coated PC.

The impressions of the indentations are examined in SEM. The results are shown in Fig. 2a-2c for Ti-coated $\mathrm{PC}$ at $100 \mathrm{mN}, 200 \mathrm{mN}$ and $300 \mathrm{mN}$ loads, respectively. Similarly indentation impressions in TiN-coated PC at $100 \mathrm{mN}, 200 \mathrm{mN}$ and $300 \mathrm{mN}$ loads are given in Fig. 2d2f. In all sample surfaces shown in Fig. 2, the lateral cracks at contact site and radial cracks, emanating from the corners of the indenter are observed, these being more prominent in $\mathrm{Ti}$. The fracture toughness $\left(\mathrm{K}_{C}\right)$ could be calculated only at the highest load $(300 \mathrm{mN})$ for $\mathrm{Ti}$ - and TiN-coated $\mathrm{PC}$, since the radial crack length (c) could be measured only for these samples. Results are $0.087 \mathrm{MPa} \mathrm{m}^{1 / 2}$ and $0.090 \mathrm{MPa} \mathrm{m}^{1 / 2}$, respectively. These results indicate that determination of $K_{C}$ by this method is not suitable, since the load is high and the effect of the substrate is dominant. At lower loads, where the effect of substrate is eliminated, it is not possible to observe the indentation impression. However, the SEM images given in Fig. 2 reveals that the coatings follow the plastic deformation of the substrate. If the in-plane stress level in the film exceeds a critical value, cracks form at the interface between the film and the substrate, then the cracks follow the topography features in the coatings. Thus, in Ti coating the cracks propagate through the valleys between the dome-shaped features whereas in TiN coating cracks follow the valleys between vermicularlike features. Unlike TiN coating, the Ti coating shows some delamination even at low loads $(100 \mathrm{mN})$. Thus, this supports the high adhesion between the TiN coating and the substrate, provided with high energetic PLD technique giving a pseudo-diffusion interface.

\section{Conclusions}

In a coating system the topography depends highly on coating material, deposition technique and the mechanical properties of the substrate. Ti coatings show columnar growth resulting in dome shaped topography with small island like features, whereas large vermicularlike, wavy topographies (i.e. wrinkles) are seen in TiN coatings, due to the differences in their elasticity moduli and deposition techniques. Besides the coating material and method, the substrate material also influences the topography as a result of its mechanical properties. The substrate also affects the fracture behavior of the coating system. PU does not show any microcracks in indentation test owing to its high elasticity.

\section{References}

[1] Y.P. Jiao, F.Z. Cui, Biomedical Materials 2, R24 (2007).

[2] P. Roach, D. Eglin, K. Rohde, C.C. Perry, J. Mater. Sci: Mater. Med. 18, 1263 (2007).

[3] M.S. Lord, M. Foss, F. Besenbacher, Nano Today 5, 66 (2010).

[4] M.J. Dalby, M.O. Riehle, H. Johnstone, S. Affrossman, A.S.G. Curtis, Cell Biology International 28, 229 (2004).

[5] J.M. Lackner, W. Waldhauser, A. Alamanou, C. Teichert, F. Schmied, L. Major, B. Major, Bulletin of the Polish Academy of Sciences Technical Sciences 58, 281 (2010).

[6] A.R. Franco Jr., G. Pintaúde, A. Sinatora, C.E. Pinedo, A.P. Tschiptschin, Materials Research 7, 483 (2004).

[7] X. Li, B. Bhushan, Materials Characterization 48, 11 (2002).

[8] J.M. Lackner, W. Waldhauser, P. Hartmann, O. Miskovics, F. Schmied, C. Teichert, T. Schöberl, Thin Solid Films 520, 2833 (2012).

[9] A. Karimi, Y. Wang, T. Cselle, M. Morstein, Thin Solid Films 420-421, 275 (2002).

[10] G.M. Pharr, Materials Science and Engineering A 253, 151 (1998). 